\title{
LEFT SELF DISTRIBUTIVE NEAR-RINGS
}

\author{
GARY BIRKENMEIER AND HENRY HEATHERLY
}

(Received 27 April 1989)

Communicated by $\mathbf{R}$. Lidl

\begin{abstract}
This paper considers (left) near-rings which satisfy the left self distributive (LSD) identity: $a b c=$ $a b a c$. This is exactly the class of near-rings for which each left multiplication mapping, $\tau_{a}: x \rightarrow$ $a x$, is a near-ring endomorphism. Simple and subdirectly irreducible ones are classified and semidirect sum decompositions into reduced and nilpotent pieces are given. LSD near-rings with restrictive conditions on nilpotent elements or annihilating sets are considered. Type 1 prime (semiprime) ideals in an LSD near-ring are completely prime (semiprime). Further results on prime and maximal ideals are given. Numerous examples are given to illuminate the theory and to illustrate its limitations. Some analogous theory for right self distributive near-rings is given (those satisfying the identity: $a b c=a c b c$ ).
\end{abstract}

1980 Mathematics subject classification (Amer. Math. Soc.) (1985 Revision): 16 A 76.

\section{Introduction}

This paper continues our investigation of near-rings satisfying certain polynomial identities, with the main emphasis herein on the left self distributive identity:

$1.1 a b c=d b a c$.

Also playing a role in this paper are the identities:

$1.2 a b c=b a c \quad($ left permutable $)$;

$1.3 a b c=a c b \quad$ (right permutable);

$1.4 \quad a b c d=a c b d \quad$ (medial);

$1.5 a b c=a c b c \quad$ (right self distributive).

(C) 1990 Australian Mathematical Society $0263-6115 / 90 \$ A 2.00+0.00$ 
(For convenience we abbreviate left self distributive by LSD and right self distributive by RSD.) Semigroups which are LSD were investigated by Kepka [14] and LSD rings were investigated by the present authors and Kepka [7]. Previously we examined in depth the properties of rings or near-rings, satisfying one of $1.2,1.3$ or $1.4[4,5,6]$. Near-rings which are both LSD and RSD, herein called self distributive, were considered by Ferrero Cotti [12] and Scapellato [25]. (Ferraro Cotti calls these near-rings "distributive" and Scapellato calls them "auto-distributive.")

The main results of this paper are

(i) a classification of simple and of subdirectly irreducible LSD nearrings;

(ii) an investigation into the properties of LSD near-rings with various restrictions on nilpotent elements or annihilating sets;

(iii) type 1 prime (semiprime) ideals in an LSD or RSD near-ring are completely prime (semiprime); further results on prime and maximal ideals are given;

(iv) a semidirect sum decomposition for an LSD or RSD near-ring into a reduced (no nonzero nilpotent elements) piece and a nilpotent piece;

(v) examples showing the abundance of LSD near-rings, their relation to the other identities given above, and limitations on the theory.

The class of LSD near-rings is a variety (and hence is closed under the formation of subdirect products). This class is exactly the class of nearrings for which each left multiplication mapping, $\tau_{a}: x \rightarrow a x$, is a near-ring endomorphism. (The class of near-rings for which every endomorphism on the additive group is a near-ring endomorphism, considered in [11], is a proper subclass of the class of LSD near-rings.)

The reader may be as surprised as were the authors to find what a large percentage of the near-rings (without identity) given in the literature satisfy the LSD property. This is discussed in Section 2. There is an interesting contrast between the paucity of LSD rings and the abundance of LSD nearrings, especially in the subdirectly irreducible situation.

The notation and terminology used herein is that used in [19], except where otherwise noted. Let $R$ be an arbitrary (left) near-ring and let $A$ and $B$ be nonempty subsets of $R$. Then $A \cdot B=\{a b: a \in A, b \in B\}$, $[A, B]=\{a b-b a: a \in A, b \in B\}$, and $\langle A\rangle$ is the ideal generated by $A$. We use $\langle R, R\rangle$ for $\langle[R, R]\rangle$. The additive group of $R$ is denoted by $R^{+}$. By a distributive element in $R$ we mean an element $d \in R$ such that $(x+y) d=x d+y d$, for each $x, y \in R$. We use $\mathscr{D}(R), \mathscr{I}(R)$, and $\mathscr{N}(R)$ for the sets of distributive elements, idempotent elements, and nilpotent elements, respectively. If no ambiguity will result, we use $\mathscr{D}, \mathscr{I}$, 
and $\mathscr{N}$ for convenience. The additive subgroup generated by $A$ is denoted by $\mathbf{g p}(A)$. We use $\mathrm{l}_{R}(A)=\{x \in R: x A=0\}$ and $\mathbf{r}_{R}(A)=\{x \in R: A x=0\}$. If no ambiguity will result, we use $\mathrm{l}(A)$ and $\mathrm{r}(A)$. If $A \cap \mathscr{N}$ is zero or empty, we say $A$ is reduced, and if $A \subseteq \mathscr{I}$ we say $A$ is Boolean. The set $A$ is said to be permutable if the elements of $A$ satisfy both 1.2 and 1.3. The commutator subgroup of $R^{+}$is denoted by $R^{\prime}$. The term semidirect sum will be taken in the additive group sense; so $A+B$ is a semidirect sum if $A$ and $B$ are subgroups of $R^{+}$, one of them is normal in $R^{+}$, and $A \cap B=0$.

An ideal $P$ in $R$ is said to be completely prime (completely semiprime) if $a, b \in R$ and $a b \in P$ implies $a \in P$ or $b \in P \quad\left(a^{2} \in P\right.$ implies $\left.a \in P\right)$. Following Ramakotaiah and Rao [23], we say an ideal $P$ is a type 1 prime (type 1 semiprime) ideal if $a R b \subseteq P$ implies $a \in P$ or $b \in P$ (aRa $\subseteq P$ implies $a \in P$ ).

\section{Examples and basic results}

Examples of LSD near-rings abound. Their abundance and wide scope were surprising to us. In this section we give some motivating examples and indicate their abundance in some of the standard sources of near-rings. Since the class of LSD near-rings is a variety (and hence is closed under the formation of subdirect products), further examples immediately arise from the ones explicitly given here. (Examples of LSD rings can be found in [7].)

EXAMPLE 2.1. (Malone's trivial near-rings [16].) These are the near-rings $R$ such that either (i) $0 \cdot R=R$ or (ii) $0 \cdot R \neq R$ and every element is either a left identity or a left annihilator of $R$. These near-rings are always LSD and left permutable. If $0 \cdot R \neq R$ and $R^{2} \neq 0$, then $R$ cannot be RSD or right permutable. It is possible for a Malone trivial near-ring $R$, with $R^{2} \neq 0$, to be d.g. and even distributive $[18,29]$.

While Malone's trivial near-rings are in some sense just that-trivial-we discussed them here because they play a key role in classifying LSD nearrings.

A perusal of the standard lists of examples of near-rings given via Cayley tables, Clay representations, or Cayley tables for generating sets $[2,9,21,22$, 30] reveals a considerable number of these near-rings are LSD. This is due only in part to the presence of Malone trivial ones. We next give a brief summary of our findings from said lists.

SUMmaRY 2.2. (i) On the Klein four group there are twenty-three nonisomorphic near-rings [9]. Fifteen of these are LSD, of which five are Malone trivial. 
(ii) On $C_{6}$ there are sixty non-isomorphic near-rings [9]. Forty-seven of these are LSD, of which twenty-one are Malone trivial.

(iii) On $S_{3}$ there are thirty-nine non-isomorphic near-rings [9]. Twentynine are LSD, of which thirteen are Malone trivial.

(iv) On the dihedral group of order eight there are twenty non-isomorphic d.g. near-rings [29]. Eighteen of these are LSD, of which three are Malone trivial.

(v) On the infinite dihedral group there are six non-isomorphic d.g. nearrings [17]. All of these are LSD and three of them are Malone trivial.

Other LSD near-rings which are not Malone trivial were found on dihedral groups of order $4 n$, generalized quaternion groups, the alternating group of degree four (Alt 4), and various cyclic groups. Some of the calculations were carried out with the aid of an IBM 3090 . Examples of particular interest will be mentioned in the sequel as it becomes appropriate.

EXAMPle 2.3. Let $(R,+, \cdot)$ be a Boolean ring, $M$ a left $R$-module, and $f: M \rightarrow R, h: M \rightarrow M R$-homomorphisms satisfying $f h=f$ and $h^{2}=h$. Define $a * b=f(a) h(b)$ for each $a, b \in M$. Then $(R,+, *)$ is a (left) near-ring which is LSD and left permutable.

A concrete realization of this occurs when $M=M_{n}(R)$, the full set of $n$ by $n$ matrices over $R, n>1$, and $f(\alpha)=\operatorname{det} \alpha, h=1_{M}$. Then $\alpha \in M$ is nilpotent in $(M, *)$ if and only if $\operatorname{det} \alpha=0$ and each nonzero nilpotent element has index two. Next suppose $R$ has unity; then the left identity elements in $(M, *)$ are exactly the matrices with determinant one. Also, $(M, *)$ is neither right permutable nor right self distributive. Using $R=Z_{2}$ we have an example where the set of nilpotent elements of $(M,+, *)$ is not closed under addition.

Other interesting realizations of the general construction arise by using $M=M_{n}(R)$, the same $f$, and $h$ one of the following:

(i) $h\left(a_{i j}\right)=\left(a_{i j}^{\prime}\right)$, where $a_{i j}^{\prime}=0$ if $i \neq j$ and $a_{j j}^{\prime}=a_{j j}$ (the diagonalizer mapping);

(ii) $h\left(a_{i j}\right)=\left(b_{i j}\right)$, where $b_{i j}=0$ if $i>j$ and $b_{i j}=a_{i j}$ if $i \leq j$ (the upper triangularizer mapping);

(iii) $h\left(a_{i j}\right)=\left(c_{i j}\right)$, where $c_{i k}=a_{i k}, i=1, \ldots, n$, and $c_{i j}=0$ if $j \neq k$, where $k$ is fixed.

(More generally, $h$ could be any projection mapping of the space $M_{n}(R)$ onto a subspace of $M_{n}(R)$.)

The above construction is a special case of a general one given in [3, 4].

EXAMPLE 2.4. (Affine near-rings $[10,13]$.) Let $(S,+, \cdot)$ be a ring and $M$ a right $S$-module. Let $R=S \times M$ and define $(\alpha, s) \odot(\beta, t)=(\alpha \beta, s \beta+t)$. Then $(R,+, \odot)$ is a (left) near-ring, the abstract affine near-ring induced by 
$S$ and $M$. If $S$ is an RSD ring and $M S^{2}=0$, then $R$ is a RSD nearring. If $M S=0$ and $S$ is medial, left permutable, or $\mathrm{LSD}$, then $(R, \odot)$ is medial, left permutable, or LSD respectively. Since every LSD ring is left permutable [7], if $S$ is LSD and $M S=0$, then $(R, \odot)$ is a left permutable and LSD.

EXAMPLE 2.5. Let $(G,+)$ be a group (not necessarily abelian) and let $h$ be any idempotent endomorphism on $(G,+)$. Define $a \otimes b=h(b)$, for each $a, b \in G$. Then $(G,+, \otimes)$ is an LSD and RSD near-ring.

EXAMPLE 2.6. (Clay construction [9].) Let $(G,+)$ be a group (not necessarily abelian) and let $f: G \rightarrow \operatorname{End}(G,+)$. Define $a * b=f(a)(b)$, for each $a, b \in G$. Then $(G, *)$ is associative and $(G,+, *)$ is a near-ring if and only if $f(a) \circ f(b)=f(a * b)$ [9, Theorem 1.2]. The near-ring $(G,+, *)$ is LSD if $f(a) \circ f(b)=f(a) \circ f(b) \circ f(a)$, for each $a, b \in G$.

EXAMPLE 2.7. Let $F$ be a free near-ring [22] and $J$ the ideal of $F$ generated (as an ideal) by $\{a b c-a b a c: a, b, c \in F\}$. Then $F / J$ is an LSD near-ring.

Often in this paper we will make use of the fact that for an arbitrary nearring $R, \mathscr{N}(R) \subseteq \mathbf{r}_{R}(0)$. (To see this, take $x^{n}=0$ and then observe that $0 x=x^{n} \cdot x=x \cdot x^{n}=x 0=0$.)

LEMMA 2.8. Let $R$ be an LSD near-ring such that $a, b, c \in R$ and $X$ is a nonempty subset of $R$.

(i) $a b c=a b a^{n} c=a b^{n} c$ for $n \geq 1$. Hence $a^{3}=a^{n} \in \mathscr{I}$ for $n \geq 3$ and if $a b c=0$, then $b a c=0$.

(ii) $R \mathscr{I} \subseteq \mathscr{I}$ and $R \mathscr{N} \subseteq \mathscr{N}$, and hence $\mathbf{g p}(\mathscr{I})$ and $\mathbf{g p}(\mathscr{N})$ are left $R$-subgroups.

(iii) $\mathscr{N} R^{2}=R \mathscr{N} R=0 \cdot R$, and hence $\mathscr{N}^{\mathscr{J}}=0 \cdot R$.

(iv) $R$ has the strong IFP property. Hence $\mathbf{r}(x)$ is an ideal and $\mathbf{r}(X) \subseteq$ $\mathbf{r}(X R), R / \mathbf{r}(a) \cong a R$ as near-rings, and $\mathbf{l}(X)=\mathbf{l}(\langle X\rangle)$.

(v) If $X^{2} a=0$, then $a X R=0 \cdot R$. Thus, if $R$ is zero symmetric, then $\mathbf{r}\left(X^{2}\right) \subseteq \mathbf{l}(X R) \subseteq \mathbf{l}\left(X^{2}\right)$.

(vi) $(a b c-b a c)^{2}=0$. Hence $\operatorname{gp}(X R \cup \mathscr{N})$ is a left $R$-subgroup.

(vii) $R=c b R+\mathbf{r}(c b)$ is a semidirect sum.

Proof. Parts (i)-(v) are straightforward applications of the definition of an LSD near-ring. For part (vi), consider $(a b c-b a c)^{2}=(a b c-b a c) a b c-$ $(a b c-b a c) b a c=(a b c-b a c)[a b(a b c-b a c) c-b a(a b c-b a c) c]=(a b c-$ $b a c)[0 c-0 c]=0$. For part (vii), let $x \in R$; then $x=c b x-c b x+x$; but $c b(-c b x+x)=0$. Therefore, $-c b x+x \in \mathbf{r}(c b)$. Assume $c b a \in$ $c b R \cap \mathbf{r}(c b)$; then $c b a=c b c b a=c b(c b a)=0$. Therefore, $R=c b R+\mathbf{r}(c b)$ is a semidirect sum. 
LEMMA 2.9. Let $R$ be an LSD near-ring such that $a, b \in R$ and $x$, $y \in \mathbf{r}(0)$.

(i) $a-a^{2} \in \mathbf{r}(0)$.

(ii) $a\left(x-x^{2}\right) y=0=\left(x-x^{2}\right) a y=a\left(x-x^{3}\right) y$.

(iii) $(a b x-a x b x)^{2}=0$.

(iv) $b=k+e+z$ where $k \in \mathscr{N}, e=e^{2} \in \mathbf{r}(0)$, and $z \in 0 \cdot R$.

Proof. (i) Consider $0\left(a-a^{2}\right)=0 a-0 a 0 a=0 a-0 a=0$.

(ii) Consider $\left(x-x^{2}\right) x^{2}=\left(x-x^{2}\right) x^{3}\left(x-x^{2}\right) x=0$. Then $\left(x-x^{2}\right)^{3}=$ $\left[\left(x-x^{2}\right) x-\left(x-x^{2}\right) x^{2}\right]\left(x-x^{2}\right)=\left(x-x^{2}\right) x\left(x-x^{2}\right)=\left(x-x^{2}\right) x^{3}\left(x-x^{2}\right)=0$. Hence $x-x^{2} \in \mathscr{N}$. By Lemma 2.8(i), $a\left(x-x^{2}\right) y=0=\left(x-x^{2}\right) a y$. By a similar argument, $a\left(x-x^{3}\right) y=0$.

(iii) $(a b x-a x b x)^{2}=(a b x-a x b x) a b x-(a b x-a x b x) a x b x=(a b x-$ $a x b x)[a b(a b x-a x b x) x-a x b(a b x-a x b x) x]=(a b x-a x b x)[(a b x-$ $\left.\left.a b x^{2}\right) x-0 x\right]=(a b x-a x b x)\left[a b\left(x-x^{2}\right) x\right]=0$ (by part ii).

(iv) There exist $x \in \mathbf{r}(0)$ and $z \in 0 \cdot R$ such that $b=x+z$. By (ii), $\left(x-x^{3}\right)^{3}=0$. Hence $x=k+x^{3}$, where $k \in \mathscr{N}$ and $x^{3}=e \in \mathscr{I}$.

LEMMA 2.10. Let $B$ be a subset of an LSD near-ring $R$ such that $B \mathscr{N} \subseteq$ $B$. If $B \cap \mathscr{N}=0$, then $B \cdot\langle\mathcal{N}\rangle=0=B \cap\langle\mathcal{N}\rangle$. In particular, $\langle\mathcal{N}\rangle$ contains no nonzero reduced right $R$-subgroup and no nonzero reduced right ideal; if $\mathscr{I} \cap\langle\mathcal{N}\rangle=0$ or $\mathscr{S} \mathscr{N}=0$, then $\mathscr{N}=\langle\mathscr{N}\rangle$.

Proof. Since $B \mathscr{N} \subseteq \mathscr{N}$, we have $B \mathscr{N} \subseteq B \cap \mathscr{N}=0$. Using left self distributivity gives $B \cdot\langle\mathscr{N}\rangle=0$. Also, $(B \cap\langle\mathscr{N}\rangle)^{2} \subseteq B \cdot\langle\mathcal{N}\rangle=0$; so $B \cap\langle\mathscr{N}\rangle \subseteq B \cap \mathscr{N}=0$. If $X$ is a reduced right ideal of $R$, then since $\mathscr{N} \subseteq \mathbf{r}(0)$ we have $X \mathscr{N} \subseteq X \cap \mathscr{N}=0$. Similarly, if $X$ is a reduced right $R$ subgroup. So $\langle\mathscr{N}\rangle$ contains no nonzero reduced right ideals and no nonzero reduced right $R$-subgroups. Finally, if $\mathscr{I N}=0$, then $\mathscr{I} \cdot\langle\mathscr{N}\rangle=0$. Since for each $x \in\langle\mathcal{N}\rangle, x^{3}$ is idempotent, we have $\langle\mathscr{N}\rangle=\mathscr{N}$.

A zero symmetric LSD near-ring can contain a reduced left ideal in the ideal generated by its nilpotents. (See $\left[9\right.$, no. 22 on $\left.S_{3}\right]$.)

Proposition 2.11. Let $R$ be an LSD near-ring and suppose $e \in \mathscr{J}, e \neq$ 0 . Then $\mathbf{r}(e)=\mathbf{r}(e R)$ and (eRe, $)$ is an idempotent, $L S D$ semigroup with $e$ as the two-sided identity. If $e \in \mathscr{D}(R)$, then $e R e$ is a Boolean ring, and $\mathbf{l}(e)=\mathbf{l}(R e)$ is both a left ideal and a right $R$-subgroup. If $e$ is central, then $e R$ is a Boolean ring and $R^{\prime} \cup\langle R, R\rangle \subseteq \mathbf{r}(e)=\mathbf{l}(e)$. 
Proof. If $x \in \mathbf{r}(e)$, then $e R x=e \operatorname{Rex}=0$; so $\mathbf{r}(e)=\mathbf{r}(e R)$. Since $e$ is a two-sided identity in $(e \operatorname{Re}, \cdot)$, this LSD semigroup is idempotent. If $e \in \mathscr{D}$, then $e R e$ is a Boolean, LSD near-ring and hence is reduced, which forces both its additive commutator subgroup and $\langle e R e, e R e\rangle$ to be zero. Thus $e R e$ is a ring. If $y e=0$, then $y R e=y R y e=0$ and hence $1(e)=1(R e)$. Finally, if $e$ is central, then $e R=e R e$ and hence $R / \mathbf{r}(e) \cong e R$, which yields $R^{\prime} \subseteq \mathbf{r}(e)$ and $\langle R, R\rangle \subseteq \mathbf{r}(e)$.

Proposition 2.12. Let $R$ be an LSD near-ring and $K=\left\{y-y^{2}: y \in\right.$ $\mathbf{r}(0)\}$. Then $\langle K\rangle$ is completely semiprime and $\langle\mathscr{N}\rangle=\langle K\rangle$.

Proof. Let $x \in R$ such that $x^{2} \in\langle K\rangle$. Let $x=b+z$ where $b \in \mathbf{r}(0)$ and $z \in 0 \cdot R$. Then $x^{2}=(b+z) b+z$. By Lemmas 2.9(i) and 2.8(iv), $\langle K\rangle \subseteq \mathbf{r}(0)$ and $\mathbf{r}(0)$ is an ideal. Hence $z=0$ and $x \in \mathbf{r}(0)$. Thus $x-x^{2} \in K$ and hence $x \in\langle K\rangle$. Therefore, $\langle K\rangle$ is completely semiprime. Consequently, $\langle\mathcal{N}\rangle \subseteq\langle K\rangle$. By Lemma 2.9(ii), $\langle K\rangle=\langle\mathscr{N}\rangle$.

Note that in general, $y-y^{2} \notin\langle\mathcal{N}\rangle$. For example, let $R$ be the LSD reduced near-ring defined in $\left[9\right.$, no. 31 on $\left.S_{3}\right]$, then $b-b^{2}=x \notin \mathscr{N}=0$.

COROLlary 2.13. Let $R$ be a zero symmetric LSD near-ring. Then $R /\langle\mathscr{N}\rangle$ is Boolean.

In the remaining results of this section we consider RSD near-rings. Example 2.4 (with $M=S, S^{3}=0$, and $S^{3}=0$, and nos. 11 and 13 on Klein four [9] are RSD near-rings which are not LSD.

LEMMA 2.14. Let $R$ be an RSD zero symmetric near-ring such that $a, b$, $c \in R$ and $X$ be a nonempty subset of $R$.

(i) $a b c=a b a^{n} c=a b^{n} c$ for $n \geq 1$. Hence $a^{3}=a^{n} \in \mathscr{I}$ for $n \geq 3$ and if $a b c=0$, then $a c b=0$.

(ii) $\mathscr{I} R=\mathscr{I}$ and $\mathscr{N} R \cup R \mathscr{N} \subseteq \mathscr{N}$.

(iii) $R \mathscr{N} R=R^{2} \mathscr{N}=0$; hence $\mathscr{I N}=0$.

(iv) $R$ has the strong IFP property. Hence $\mathbf{r}(X)$ is an ideal of $R$ and $\mathbf{r}(X) \subseteq \mathbf{r}(X R), R / \mathbf{r}(a) \cong a R$ as near-rings, and $\mathbf{l}(X)=\mathbf{l}(\langle X\rangle)$.

(v) If $a X^{2}=0$, then $R X a=0$; hence $\mathbf{l}\left(X^{2}\right) \subseteq \mathbf{r}(R X) \subseteq \mathbf{r}\left(X^{2}\right)$.

(vi) $\left(x-x^{2}\right)^{3}=0=\left(x-x^{3}\right)^{3}$.

(vii) $b=k+e$ where $k \in \mathscr{N}$ and $e \in \mathscr{F}$.

Proof. The proof is similar to that used in the LSD case. 
Proposition 2.15. Let $R$ be a near-ring.

(i) If $R$ is $L S D$ and has a right identity, then $R$ is a Boolean ring.

(ii) If $R$ is RSD and has a left identity, then $R$ is a Boolean near-ring. If the identity is two-sided, then $R$ is a Boolean ring.

Proof. (i) An LSD semigroup with right identity is idempotent. So $R$ is a Boolean near-ring with right identity. Ligh [15] has shown such near-rings are rings.

(ii) For each $r \in R$, if $e$ is a left identity, then $r=e r=e r e r=r^{2}$. If $e$ is two-sided use Ligh's result mentioned in (i).

An RSD near-ring with left identity need not be a ring. Either the nearring composed of all left identities or the zero symmetric one with all nonzero elements being left identities on any nonabelian additive group serve as examples.

The following result generalizes [25, Theorem 2].

Proposition 2.16. Let $R$ be an RSD near-ring. Then $\mathcal{N}$ is a right ideal. Furthermore

(i) if $R$ is zero symmetric, then $\mathscr{N}$ is an ideal and $R / \mathscr{N}$ is a Boolean near-ring, and

(ii) if $R$ is $L S D$, then $\mathcal{N}$ is an ideal.

Proof. Let $a, b \in \mathscr{N}$ and $x, y \in R$. Then $(a-b)^{3}=(a-b)^{2} a-(a-$ $b)^{2} b=(a-b) a^{3}(a-b) a-(a-b) b^{3}(a-b) b=0$. Hence $\mathcal{N}$ is a group. Now observe that $(x+a-x)^{2} a=(x+a-x) a^{3}(x+a-x) a=0$. Hence $(x+a-x)^{3}=0$. Thus $\mathcal{N}$ is a normal subgroup. Let $s=(x+a) y-x y$ and $y=c+k$, where $c \in \mathbf{r}(0)$ and $k \in 0 \cdot R$. Then $s^{2}=s(x+a) c+k-(s x c+k)=$ $s c^{2}(x+a) c-s c^{2} x c=\left(c^{2} x+c^{2} a\right) c-s c^{2} x c=s c^{2} x c-s c^{2} x c=0$. Therefore, $\mathscr{N}$ is a right ideal. Part (i) follows from Lemma 2.14(ii). For part (ii) Observe that $(x a)^{3}=x a^{3}=0$.

In general, $\mathscr{N}$ will not be an ideal in an RSD near-ring. Recall Example 2.4 and assume $M S^{2}=0$. Then $R$ is $\operatorname{RSD}$ and $(a, m)^{3}=0$ if and only if $m \alpha+m=0$. But $m \alpha \alpha+m \alpha=m \alpha=0$. Hence $m=0$. Thus $\mathscr{N}(R)=\{(\alpha, 0): \alpha \in S\}$. If $M S \neq 0$, then $\mathscr{N}(R)$ is not a left ideal.

PROPOSITION 2.17. Let $R$ be an LSD or an RSD zero symmetric near-ring and $P$ an ideal of $R$. Then $P$ is completely prime (completely semiprime) if and only if $P$ is type 1 prime (type 1 semiprime). 
Proof. Note that for any near-ring a completely prime (completely semiprime) ideal is a type 1 prime (type 1 semiprime) ideal. Assume $R$ is LSD, $a b \in P, x \in R$, and $P$ is a type 1 prime ideal. Then $a x b=a x a b \in P$. Hence $a R b \subseteq P$. So $P$ is a completely prime ideal. The other cases are similar.

We observe that, in general, for LSD near-rings an ideal may be prime but not necessarily completely prime. For example, no. 30 on $S_{3}$ in [9] is LSD, RSD, and prime, but not an integral near-ring.

\section{Simple LSD near-rings}

The following lemma will be useful in this section, as well as subsequently.

LEMMA 3.1. Let $R$ be an LSD near-ring with $\mathbf{r}(R)=0$. If $a, b \in R$, then

(i) $a b=a^{2} b$ and $a^{2} \in \mathscr{I}$;

(ii) $\mathscr{N} \cdot R=0 \cdot R$ (hence $\left.\mathscr{N}^{2}=0\right)$.

Proof. This follows from Lemma 2.8(i), (iii).

THeOREM 3.2. Let $R$ be a simple LSD near-ring. Then either (exclusively)

(i) every element of $R$ is a left identity and $R^{+}$is a simple group, or

(ii) $R$ is zero symmetric and every element of $R$ is either a left identity or a left annihilator of $R$.

Proof. If $\mathbf{r}(0)=0$, then every element of $R$ is a left identity. In this case each normal subgroup of $R^{+}$is an ideal of $R$, so $R^{+}$is simple.

Consider $\mathbf{r}(0)=R$. If $\mathbf{r}(R)=R$, then $R^{2}=0$ and hence $R^{+}$is a simple group. If $\mathbf{r}(R)=0$, then for each nonzero $x \in R$, either the idempotent $x^{2}$ is a left identity, or $\mathbf{r}\left(x^{2}\right) \neq 0$ and $0=x^{2} R=x R$, from Lemma 3.1(i).

Thus all simple LSD near-rings are Malone trivial ones. (But not all Malone trivial near-rings are simple.)

CoROLlaRY 3.3. If $R$ is a simple, LSD near-ring, $R^{2} \neq 0$, and $\mathscr{D}(R) \neq 0$, then $R \cong Z_{2}$.

Proof. Checking cases shows that $L+L \subseteq A, L+A \subseteq L, A+L \subseteq L$, and $A+A \subseteq A$, where $L$ is the set of left identity elements and $A$ is the set of left annihilating elements. It follows that $\mathrm{l}(R)$ is an ideal and $\mathscr{N}=\mathbf{l}(R)$. 
Simplicity and $R^{2} \neq 0$ yields that every nonzero element is a left identity. Thus the sum of any two nonzero elements is zero, which forces $|R|=2$ and $R \cong Z_{2}$.

COROLlary 3.4. Let $R$ be a self distributive near-ring. If $R$ is simple, then either

(i) $0 \cdot R=R$,

(ii) $R^{2}=0$ and $R^{+}$is a simple group; or

(iii) $R$ is zero symmetric and each nonzero element of $R$ is a left identity.

Proof. If $0 \cdot R \neq R$ and $R^{2} \neq 0$, then $0 \cdot R=0$ and $R$ contains a left identity element $b$. If $c \in \mathscr{N}$, then $0=c^{2}=b c b c=b b c=c$.

Each of the three types given in this corollary are indeed self distributive and simple. For type (iii), $R^{+}$can be any group.

The following is an extension of [12, Theorem 7] and has that result as an immediate corollary.

Proposition 3.5. Let $R$ be a zero symmetric, LSD near-ring with DCC on right ideals. If either of the radicals $J_{1}(R)$ or $J_{2}(R)$ are zero, then $R$ is a finite direct sum of simple near-rings of the type given in Theorem 3.2(ii) and $R$ has a left identity. Consequently, $R$ is left permutable and $R$ is right permutable if and only if $R$ is a Boolean ring.

Proof. The standard structure theory ([19, Chapter 7] or [22, Chapter 5]) yields the decomposition of $R$ into a direct sum of a finite number of simple, zero symmetric near-rings with left identity. Since each summand is LSD, we have the conditions necessary to invoke Theorem 3.2. Each summand is left permutable and hence $R$ is also. Similarly, a summand is right permutable if and only if it is isomorphic to $Z_{2}$.

We leave as open the question of whether $J_{1}(R)$ or $J_{2}(R)$ zero in Proposition 3.5 can be replaced by " $R$ has zero nil radical."

COROLLARY 3.6. Let $R$ be an LSD near-ring with DCC on right ideals and with either $J_{1}(R)$ or $J_{2}(R)$ zero. If $R$ is d.g., then $R$ is a finite Boolean ring.

Proof. Proceed as in the proof of Proposition 3.5. Each summand will contain a nonzero distributive element and hence be isomorphic to $Z_{2}$.

Proposition 3.8. Let $R$ be a zero symmetric, $L S D$ near-ring with $D$. $C$. C. on right ideals. If $R$ is (von Neumann) regular, then $R$ is a finite direct sum of near-rings which have every nonzero element a left identity. 
Proof. First observe that $R$ is a finite direct sum of simple, LSD, regular near-rings. From Theorem 3.2(ii) these are the ones with every nonzero element a left identity. (There can be no nonzero left annihilating elements in a regular near-ring.)

PROPOSITION 3.9. If $M$ is a maximal ideal in an LSD near-ring $R$, then $M$ is maximal as a right ideal of $R$.

Proof. By Theorem 3.2 every element of $R / M$ is either a left identity or a left annihilator. If $x+M$ is the former, then for each $r \in R, x r=r+m$, for some $m \in M$; if $x+M$ is the latter, then $x r \in M$ for each $r \in R$. Suppose $S$ is a right ideal of $R$ and $M \subseteq S$. Then for each $s \in S, x \in R$ we have $x s=s+m$, where $m \in M$, or $x s \in M$. In either case, $x s \in S$ and hence $S$ is an ideal of $R$.

\section{Reduced LSD near-rings and allied topics}

In this section we consider LSD near-rings under various conditions that restrict products from being zero (for example, reduced, $l(R)=0, \mathbf{r}(R)=0$, integral). This leads on the one hand to subdirect product classifications and on the other to some interesting properties of prime ideals.

Proposition 4.1. If $R$ is a reduced LSD near-ring, then $R$ is left permutable and $a^{2}$ is a nonzero idempotent for each $a \in R$.

Proof. Left permutability follows from Lemma 2.8(vi). That $a^{2}$ is a nonzero idempotent follows from Lemma 3.1.

Reduced LSD near-rings need not be Boolean nor self distributive, even if $R$ is subdirectly irreducible. There are several examples of this among the near-rings on $S_{3}$ [9]. A dramatic example is afforded by [9, no. 12], which is reduced, LSD, left permutable, and subdirectly irreducible, but not self distributive, Boolean, integral nor right permutable. The situation greatly improves in the zero symmetric case. A reduced, zero symmetric near-ring is isomorphic to a subdirect product of integral near-rings [22, 9.38], a situation which need not hold if the zero symmetric hypothesis is dropped, as the example just mentioned illustrates.

Proposition 4.2. Let $R$ be an LSD near-ring.

(i) If $R$ is a reduced, zero symmetric near-ring, then $R$ is Boolean.

(ii) $R$ is regular if and only if $R$ is Boolean. 
Proof. Part (i) follows from Lemma 2.9(ii) and (iii). For part (ii), assume $R$ is regular and let $x \in R$. Then there exists $a \in R$ such that $x=x a x=$ $x a x x=x^{2}$. The converse is obvious.

Boolean, left permutable near-rings were called “ $\beta$-near-rings” by Ligh who characterized them in terms of subdirectly irreducible components [15]. (Earlier Subrahmanyan [28] considered Boolean, left permutable near-rings with abelian additive group, which he called "Boolean semirings.") It is immediate that a $\beta$-near-ring is LSD. Thus in the class of zero symmetric nearrings the subclass of $\beta$-near-rings and the subclass of reduced LSD near-rings coincide. Scapellato showed that the class of $\beta$-near-rings coincides with the class of self distributive, Boolean near-rings and he gave a characterization of these near-rings in terms of subdirect products of subdirectly irreducible ones [25].

For Boolean near-rings several classes specified by various identities coalesce.

Proposition 4.3. Let $R$ be a Boolean near-ring. The following are equivalent:

(i) $R$ is $L S D$;

(ii) $R$ is left permutable;

(iii) $R$ is medial;

(iv) $R$ is self distributive.

Proof. Since Boolean rings are reduced, (i) implies (ii). If $R$ is medial, then for each $a, b, c \in R, a b c=a b c c=a c b c$; so (iii) implies (iv). The other implications are immediate.

LEMMA 4.4. If $R$ is an LSD near-ring and $c \in \mathbf{r}_{R}(0), z \in 0 \cdot R$, then $z c=0$.

Proof. $z c=0 z c=0 z 0 c=0 z 0=0$.

PROPOSITION 4.5. If $R$ is an integral, LSD near-ring, then either $0 \cdot R=R$, or $R$ is zero symmetric and every nonzero element is a left identity. In the latter case either $\mathscr{D}(R)=0$ or $R \cong Z_{2}$.

Proof. By Lemma 4.4 either $r_{R}(0)=0$ (and hence $0 \cdot R=R$ ), or $0 \cdot R=$ 0 . In the latter case, by Proposition 4.2, we have $R$ is Boolean. Since for each nonzero $x$ in $R, \mathbf{r}(x)=0$ and $x$ is idempotent, we have $x$ is a left identity. Furthermore, if $\mathscr{D}(R) \neq 0$, then every nonzero element being a left identity forces $R$ to have exactly two elements and consequently $R \cong Z_{2}$. 
THEOREM 4.6. If $P$ is a type 1 prime ideal of an LSD near-ring $R$, then every normal right $R$-subgroup of $R$ which contains $P$ is an ideal. If $P$ is also a maximal ideal, then $P$ is maximal as a normal right $R$-subgroup of $R$.

Proof. From Proposition 2.17, $P$ is completely prime and hence $R / P$ is integral. By Proposition 4.5, if $R$ is zero symmetric then every nonzero element of $R / P$ is a left identity. Thus, for each $x, r \in R, x \neq 0$, we have $x r=r+p$, where $p \in P$. Let $S$ be a normal right $R$-subgroup such that $P \subseteq S$. For each $s \in S$ and any $r_{1}, r_{2} \in R$, if $r_{1} \in S$ we have $\left(s+r_{1}\right) r_{2}-r_{1} r_{2} \in S$, because $S$ is a right $R$-subgroup; and if $r_{1} \notin$ $S$, then $\left(s+r_{1}\right) r_{2}=r_{2}+p_{1}$ and $r_{1} r_{2}=r_{2}+p_{2}$, where $p_{1}, p_{2} \in S$, so $\left(s+r_{1}\right) r_{2}-r_{1} r_{2}=r_{2}+p_{1}-\left(r_{2}+p_{2}\right)=r_{2}-r_{2}+p_{3}$, where $p_{3} \in P$. Since $P \subseteq S$, we have $\left(s+r_{1}\right) r_{2}-r_{1} r_{2} \in S$ and hence $S$ is a right ideal. An argument similar to that in the proof of Proposition 3.9 gives that this right ideal is an ideal. If $R$ is also a maximal ideal, then $P=S$ and $P$ is maximal as a normal $R$-subgroup.

From Lemma 4.4 we see that if $0 \cdot R \neq 0$ and $P$ is a type 1 prime ideal such that $0 \cdot P=0$, then $\mathbf{r}(0)=P$.

Proposition 4.7. Let $R$ be a zero symmetric $L S D$ near-ring and $P$ be a type 1 prime ideal of $R$ such that there exists $d \in \mathscr{D}(R)$ with $d \notin P$. Then $R / P \cong Z_{2}, P$ is maximal as a subgroup of $R^{+}$, and $\left\langle R^{\prime}\right\rangle \cup\langle R, R\rangle \subseteq P$.

Proof. $\mathscr{D}(R / P) \neq 0$. So Proposition 4.5 yields $R / P \cong Z_{2}$. The rest of the conclusion is immediate.

Note that Proposition 4.7 holds if $R$ is d.g.

Ramakotaiah and Rao [23, Corollary 3.6] observed that in an IFP, zero symmetric near-ring, every type 1 prime ideal contains the set of nilpotent elements. From Proposition 2.17 we see that for any LSD near-ring $R$ the nilpotent elements are contained in each type 1 semiprime ideal. Furthermore, if $S$ is a type 1 semiprime ideal of $R$, then $R / S$ is reduced and left permutable; if $R$ is also zero symmetric, then $R / S$ is Boolean and RSD.

The intersection of all type 1 prime ideals of a near-ring $R$ will be denoted by $P_{1}(R)$ as in [23].

Proposition 4.8. Let $R$ be an LSD near-ring. If $P_{1}(R)=0$ then $R$ is isomorphic to a subdirect product of integral near-rings. If $R$ is also d.g., then $R$ is a Boolean ring.

Proof. Standard subdirect product methods together with $R / P$ is integral for each type 1 prime ideal $P$ and Proposition 4.5 give the desired result. 
Proposition 4.9. Let $R$ be a reduced LSD near-ring. Then $R=0 \cdot R+$ $\mathbf{r}(0)$, as a semidirect sum of a two-sided $R$-subgroup and an ideal, such that ideal $\mathbf{r}(0)$ is isomorphic to a subdirect product of near-rings each of which has every nonzero element a left identity. If $R$ is also d.g., then $R$ is a Boolean ring.

Proof. The Peirce decomposition with the idempotent zero gives $R=$ $0 \cdot R+\mathbf{r}(0)$. Every zero symmetric, reduced near-ring is isomorphic to a subdirect product of integral near-rings $[22,9.36]$. Proposition 4.5 then gives the desired results.

Proposition 4.10. Let $R$ be a near-ring. Then $R$ is $L S D$ and $1(R)=0$ if and only if $R$ is zero symmetric, Boolean, self distributive, and left permutable.

Proof. Assume $R$ is LSD and $\mathrm{l}(R)=0$. From the definition of $1(R)=0$ we have $0 \cdot R=0$. If $x \in \mathscr{N}$, then $x R^{2}=0$; so $x=0$. By Propositions 4.2 and 4.3 we have that $R$ is Boolean, self distributive, and left permutable. The converse follows from Proposition 4.3.

It is possible for an LSD near-ring with zero left annihilator to be not right permutable. The zero symmetric near-ring all of whose nonzero elements are left identities is an example of such (on any group with more than one element).

For any LSD near-ring $R$, "reduced" is a weaker condition than $\mathrm{l}(R)=0$. Examples of this behavior abound. (For example, no. 10 on $S_{3}$ in [9] is reduced, LSD, left permutable, not RSD, not right permutable, not Boolean and has empty left annihilator. This near-ring is also subdirectly irreducible, but not simple.)

At first though one might expect to obtain analogous results for LSD nearrings with zero right annihilator. A perusal of examples of such near-rings shows the navete of this thought, for there are LSD, zero symmetric nearrings with right annihilator zero, yet which are not medial, RSD, nor Boolean. (See $\left[9\right.$, no. 27 on $S_{3}$ ] for one which is also subdirectly irreducible and has a left identity.) Yet some things can be said for this class of near-rings, as seen in Lemma 3.1 and in the next results.

Proposition 4.11. If $R$ is a regular, right permutable near-ring, then $R$ is a reduced commutative ring.

Proof. Since $R$ is regular and zero symmetric, $\mathbf{r}(R)=0$. However, right permutability yields $R \cdot[R, R]=0$. So $R$ is commutative and hence $R \cdot R^{\prime}=0$, forcing $R^{\prime}=0$ and $R$ to be a ring. Commutative regular rings are reduced. 
Proposition 4.12. Let $R$ be an LSD near-ring with $\mathbf{r}(R)=0$. If $R$ is right permutable, then $R$ is a Boolean ring.

Proof. Right permutability implies $R \cdot\langle R, R\rangle=0$ [4]. Thus $\langle R, R\rangle=0$ and $R$ is commutative. A commutative near-ring with zero right annihilator is a ring. Thus $R$ is a self distributive ring and hence $R=\mathscr{N} \oplus B$, where $B$ is Boolean [20]. This yields $R \mathscr{N}^{2}=0$ and hence $\mathscr{N}^{2}=0$; so $R \mathscr{N}=0$, which forces $\mathscr{N}=0$.

Considerably more can be said about LSD near-rings with zero right annihilator in the d.g. case. We take up this topic and expand the view to taking a class of near-rings considerably wider than d.g. in a subsequent paper.

\section{Subdirectly irreducible}

In this section subdirectly irreducible LSD near-rings are classified. We begin with some useful general results connecting annihilating sets and left identity elements in LSD near-rings.

LeMma 5.1. Let $S$ be an LSD semigroup and $x, y \in S$. Then $x y$ is a left identity if and only if $x$ and $y$ are left identities.

Proof. Assume $x y$ is a left identity and $b \in S$. Then $y b=x y y b=$ $x y b=b=x y(x b)=x b$. The converse is obvious.

Proposition 5.2. Let $R$ be an LSD near-ring and $x, y \in R$.

(i) $\mathbf{r}(x y)=\mathbf{r}(y x)$.

(ii) If $\mathbf{r}(x y)=0$, then $x$ and $y$ are left identities.

(iii) If $x$ is not a left identity, then $0 \neq \mathbf{r}(x y) \cap y R \subseteq \mathbf{r}(x)$.

Proof. (i) Let $a \in \mathbf{r}(x y)$ and consider $y x a=y x y a=0$. Hence $\mathbf{r}(x y) \subseteq$ $\mathbf{r}(y x)$. Similarly, $\mathbf{r}(y x) \subseteq \mathbf{r}(x y)$.

(ii) Let $b \in R$. Then $x y(b-x y b)=0$. Hence $x y b=b$. By Lemma 5.1, $x$ and $y$ are left identities.

(iii) By part (ii), $0 \neq \mathbf{r}(x y)$. Let $y b \in \mathbf{r}(x y) \cap y R$. Then $0=x y(y b)=$ $x(y b)$. Hence $\mathbf{r}(x y) \cap y R \subseteq \mathbf{r}(x)$.

Proposition 5.3. Let $R$ be a LSD near-ring and $e \in R$; then the following are equivalent

(i) $e$ is a left identity; 

(ii) $\mathbf{r}\left(e^{2}\right)=0$;
(iii) $\mathbf{r}(e)=0$;
(iv) $e R=R$.

Proof. Clearly (i) implies (ii), (iii), and (iv). By Proposition 5.2, (ii) implies (i). Assume $\mathbf{r}(e)=0$. Then $e\left(e^{2}-e^{3}\right)=0$, so $e^{2}=e^{3}$. Hence $e\left(e-e^{2}\right)=0$, so $e=e^{2}$. Thus $\mathbf{r}(e)=0=\mathbf{r}\left(e^{2}\right)$. Consequently, (iii) implies (ii). Assume $e R=R$ and $e$ is not a left identity. Then $e^{2} R=R$ and there exists $0 \neq b \in \mathbf{r}(e)$. Hence there exists $x \in R$ such that $b=e^{2} x$. Consider $b=e^{2} x=e\left(e^{2} x\right)=e b=0$, a contradiction. Thus (iv) implies (i).

It is worth noting a connection between LSD near-rings and geometric near-rings, thereby linking them with non-commutative geometry in the sense of André [1].

COROLlaRY 5.4. Let $R$ be a finite, zero symmetric near-ring. Then $R$ is $L S D$ and strongly geometric if and only if $R$ has a left identity and every element is either a left identity or a left annihilator of $R$.

Proof. This result follows from Proposition 5.3 and Scapellato [26, Theorem 2] and [27, Corollary 2.6].

There are several LSD strongly geometric near-rings on $S_{3}$. Some of the simple ones are nos. 4, 5, 20,21, 22 [9], whereas no. 36 [9] is d.g. but not simple.

In the sequel $H(R)$, or where the meaning is clear just $H$, will denote the heart of the near-ring $R$ (the intersection of all nonzero ideals of $R$ ).

COROLlARY 5.5. If $R$ is an LSD subdirectly irreducible near-ring, then either $R$ has a left identity or $R H=0$.

Proof. If $R$ has no left identity, then $0 \neq H \subseteq \mathrm{r}(x)$ for all $x \in R$. Therefore, $R H=0$.

THEOREM 5.6. Let $R \neq 0$ be a LSD subdirectly irreducible near-ring. Then either (exclusively)

(i) $R \cdot H=0$ and $\mathrm{l}(R) \neq 0$,

(ii) $0 \cdot R=R$ and $H$ contains no nonzero normal subgroups of $R^{+}$other than itself, or

(iii) each element of $R$ is either a left identity for $R$ or a left annihilator for $H$, and at least one element of each type exists; either $H$ contains a left identity of $R$ and $H=\mathbf{r}(0)$, or $H^{2}=0$ and $H R=0 \cdot R$. 
Proof. If $R$ has no left identity, then $R \cdot H=0$. Hence $\mathrm{l}(R) \neq 0$, for otherwise $R$ is Boolean (Proposition 4.10).

Next assume $R$ has a left identity. If every element of $R$ is a left identity, then any normal subgroup of $R^{+}$which is contained in $H$ is an ideal of $R$; hence the only such normal subgroups are 0 and $H$. This case aside, we can consider $\mathbf{r}(0) \neq 0$ and hence $H \subseteq \mathbf{r}(0)$. Since $R$ has a left identity, $\mathbf{r}(R)=0$ and by Lemma 3.1 we have $x^{2}$ is an idempotent for each $x \in R$. If $\mathbf{r}\left(x^{2}\right)=0$, then $x$ is a left identity. Otherwise, $x H=x^{2} H=0$. If $H$ contains a left identity of $R$, then $H=\mathbf{r}(0)$; otherwise, every element in $H$ is a left annihilator of $H$ and hence $H^{2}=0$ and $H \cdot R=H R^{2}=0 \cdot R$.

Using Birkhoff's Theorem [8] we have that every LSD near-ring is isomorphic to a subdirect product of subdirectly irreducible LSD near-rings of types (i), (ii), or (iii) in Theorem 5.6. For special types of LSD near-rings we get much sharper results.

Lemma 5.7 [7]. Let $R$ be an LSD subdirectly irreducible ring. Then $R$ satisfies either

(i) $R \cong Z_{2}$,

(ii) $R^{3}=0$, or

(iii) $R \cong Z_{2}[S]$, the semigroup ring formed by $Z_{2}$ over the two element semigroup $S$ which has both elements left identities. This ring is left permutable, but is not right permutable.

Proposition 5.8. Let $R$ be an LSD subdirectly irreducible near-ring. If $R$ is right permutable, then $R$ satisfies either (exclusively).

(i) $R \cong Z_{2}$,

(ii) $R^{3}=0$, or

(iii) $R^{3} \neq 0, R \cdot H=0, H \subseteq(R, R\rangle \cap\left\langle R^{\prime}\right\rangle$, and $\mathbf{l}(R) \neq 0$.

Proof. Right permutable near-rings are zero symmetric, eliminating type (ii) in Theorem 5.6. If $R$ is of type (iii) in Theorem 5.6, then $R$ has a left identity and hence is commutative. A commutative near-ring with a left identity is a ring. The only right permutable, LSD subdirectly irreducible rings are those satisfying $R \cong Z_{2}$ or $R^{3}=0$. So now consider $R^{3} \neq 0$, $R^{\prime} \neq 0$, and $\langle R, R\rangle \neq 0$. This forces us to type (i) of Theorem 5.6 and to $H \subseteq\langle R, R\rangle \cap\left\langle R^{\prime}\right\rangle$.

Examples with all the properties of Proposition 5.8 (iii) do exist. Nearring no. 9 on $S_{3}$ in [9] is one such; it has two nonzero idempotents and is not left permutable. Near-ring no. 29 on $S_{3}$ in [9] is another such, but it is left permutable and has only one nonzero idempotent. 


\section{Decompositions}

In developing a decomposition theory for LSD near-rings, Lemma 2.9(iv) suggest that a near-ring $R$ can be split in terms of a subnear-ring generated by nilpotent elements and a subnear-ring generated by idempotent elements. We begin this section with several results which build up to an "essential decomposition" of a zero symmetric LSD near ring in terms of a Boolean near-ring and $\langle\mathcal{N}\rangle$. From there our investigations culminate in Theorem 6.8 in which a self distributive permutable near ring $R$ is characterized as a semidirect sum $R=B+\mathscr{N}$, where $B$ is a Boolean ring and $\mathscr{N}$ is an ideal. This result generalizes results of M. Petrich [20] and C. Ferrero Cotti [12].

Proposition 6.1. Let $X$ be a right ideal of an LSD near-ring $R$ and $a$, $s \in R$ such that $a^{2} \in X$. Then $-0 a+s a^{2} \in X$. If $a^{3} \in X$, then $s a^{2} \in X$.

Proof. Observe $\left(a^{2}+0\right) s-0 s=a^{2} s-0 s \in X$. Then $0 s-a^{2} s \in X$. Consider $\left[\left(0 s-a^{2} s\right)+s\right]\left(-a^{2}\right)-s\left(-a^{2}\right)=-\left(\left[\left(0 s-a^{2} s\right)+s\right] a^{2}\right)+s a^{2}=$ $-\left(\left[\left(0 s-a^{2} s\right)+s\right] a^{3}\left[\left(0 s-a^{2} s\right)+s\right] a\right)+s a^{2}=-\left(\left[\left(0 s-a^{2} s\right)+s\right]\left[0 s-a^{3} s+\right.\right.$ $\left.\left.a^{3} s\right] a\right)+s a^{2}=-\left(\left[\left(0 s-a^{2} s\right)+s\right] 0 s a\right)+s a^{2}=-0 a+s a^{2} \in X$. By substituting $a$ for $s$ we have $-0 a+a^{3} \in X$. Hence, if $a^{3} \in X$, then $-0 a \in X$ and $s a^{2} \in X$.

COROLLARY 6.2. If $X$ is a reduced right ideal of an LSD near-ring $R$ and $X \subseteq \mathbf{r}(0)$, then $X$ is an ideal of $R$.

Proof. First note that $X$ is a subnear-ring of $R$. So by Proposition 4.2, $X$ is Boolean. Proposition 6.1 then gives $R X \subseteq X$ and hence $X$ is an ideal of $R$.

We say a nonempty subset $X$ of $R$ is left (right) essential in $R$ if every nonzero left (right) ideal of $R$ has nonzero intersection with $X$.

THEOREM 6.3. Let $R$ be an LSD zero symmetric near-ring and $A$ be a left ideal of $R$ which is maximal among left ideals having zero intersection with $\langle\mathscr{N}\rangle$. Then $A$ contains every reduced right ideal of $R$. Hence $A+\langle\mathscr{N}\rangle$ is a semidirect sum which is left and right essential in $R$ and $A$ is a Boolean near-ring.

Proof. Let $X$ be a reduced right ideal of $R$. Then $X$ is an ideal of $R$ and $X+A$ is a left ideal of $R$. If $a \in A, x \in X$ are such that $x+a \in(\mathscr{N})$, then $x \cdot(x+a) \in X \cap\langle\mathscr{N}\rangle$, and $X \cap\langle\mathscr{N}\rangle=0$ by Lemma 2.10. Recalling 
from Proposition 4.2 that $X$ is Boolean, we have $0=x \cdot(x+a)=x+x a$, or $x=-x a$ and hence $X \subseteq A$.

If $L$ is a nonzero left ideal of $R$ such that $L \cap A=0$, then $A \subset L+A$ and hence $(L+A) \cap\langle\mathcal{N}\rangle \neq 0$. So there exists $a \in A, b \in\langle\mathscr{N}\rangle$, and nonzero $y \in L$ such that $y+a=b$, or $y=b-a \in\langle\mathscr{N}\rangle+A=A+\langle\mathscr{N}\rangle$. Thus $A+\langle\mathscr{N}\rangle$ is left essential in $R$. For any nonzero right ideal $W$, if $W \cap\langle\mathscr{N}\rangle=0$, then $W$ is also a left ideal and hence $W \cap(A+\langle\mathcal{N}\rangle) \neq 0$.

COROLlARY 6.4. Let $R$ be an LSD zero symmetric near-ring. There exist a unique maximal reduced ideal $M$ of $R$ which contains every reduced right ideal of $R$. Hence $M \oplus\langle\mathscr{N}\rangle$ is a direct sum which is right essential in $R$ and $M$ is a Boolean near-ring.

Proof. The proof is similar to the proof of Theorem 6.3.

LEMMA 6.5. Let $R$ be an LSD near-ring. Then $\operatorname{gp}(\mathscr{I})$ is reduced and $[\operatorname{gp}(\mathscr{I})] \mathcal{N}=0$ if and only if $R=\operatorname{gp}(\mathscr{I})+\mathscr{N}$ is a semidirect sum, where $\mathscr{N}$ is an ideal and $\mathbf{g p}(\mathscr{I})$ is a two-sided $R$-subgroup.

Proof. The result follows from Lemmas 2.8(ii), 2.9(iv), and 2.10.

Examples exhibiting the decomposition indicated in Lemma 6.5 can be found in [9, nos. $57,58,60$ on $Z_{6}$, no. 22 on Klein four, and no. 33 on $S_{3}$ ]. These examples are all self distributive left permutable, but not right permutable; number 58 on $Z_{6}$ is zero symmetric.

Proposition 6.6. Let $B$ be an LSD near-ring. The following are equivalent

(i) $\mathbf{g p}(\mathscr{I})$ is reduced, right permutable, and $[\operatorname{gp}(\mathscr{I})] \mathscr{N}=0$;

(ii) $R=\mathscr{I}+\mathscr{N}$ is a semidirect sum where $\mathscr{N}$ is an ideal, $\mathscr{I}$ is a Boolean ring and a two-sided $R$-subgroup which is contained in the center of $R$, and $R$ is zero symmetric.

Proof. Assume (i) and let $y \in 0 \cdot R$. Then $0 y=00 y=0 y 0$. Hence $R$ is zero-symmetric. By Proposition 4.12, $\mathrm{gp}(\mathscr{J})=\mathscr{I}$ is a Boolean ring. We will show that $\mathscr{I}$ is in the center of $R$. Let $x \in R$ and $s \in \mathscr{I}$. There exist $e \in \mathscr{I}$ and $k \in \mathscr{N}$ such that $x=e+k$. Since $x s \in \mathscr{I}$, then $x s=(x s) s=s(x s)=s e s=s e=s x$. The remainder of part (ii) follows from Lemma 6.5. The proof of the converse is immediate.

LEMMA 6.7. Let $R$ be a near-ring.

(i) If $R$ is permutable, then every idempotent is central.

(ii) If $R$ is $R S D$ and right permutable, then $a b c=-a b c$. 
(iii) If $R$ is LSD and right permutable, then $R$ is self-distributive.

(iv) If $R$ is a distributive near-ring and LSD (RSD), then $R$ is left permutable (right permutable) and $a b c=-a b c$.

Proof. Let $a, b, c, d \in R$ and $e \in \mathscr{I}$.

(i) Consider $e b=e e b=e b e=b e e=b e$.

(ii) Observe that $-a b c=a b(-c)=a(-c) b(-c)=-[a(-c) b c]=$ $-[a b c(-c)]=a b c c=a c b c=a b c$.

(iii) Observe that $a b c=a c b=a c a b=a c a c b=a c a b c=a c b c$.

(iv) Assume $R$ is LSD; then $-a b c=(-a) b c=(-a) b(-a) c=a b a c=$ $a b c$. The proof that $R$ is left permutable is the same as that given in [7, Proposition 1.2]. We will adapt it to the RSD condition. Now assume $R$ is RSD; then $0=a(c+d) b(c+d)-a b(c+d)=a c b d+a d b c$. Thus $a c b d=a d b c$. Now using the preceding identity and right self distributivity, we have $a b c-a c b=a c b c-a b c b=a c^{2} b c-a c b c b=a c b c^{2}-a c^{2} b=$ $a b c c-a c^{2} b=a c c b-a c^{2} b=0$.

We observe that, in general, an LSD zero symmetric near-ring may not be medial $\left[9\right.$, no. 27 on $\left.S_{3}\right]$, hence not left permutable. Surprisingly, there are self distributive, zero symmetric, medial near-rings which are neither left nor right permutable [30, nos. 80 and 132 on Alt. 4].

THEOREM 6.8. Let $R$ be a near-ring. The following are equivalent:

(i) $R$ is $L S D$, right permutable, and $\mathbf{g p}(\mathscr{I})$ is reduced;

(ii) $R$ is right permutable and $R=B+\mathscr{N}$ is a semidirect sum, where $\mathscr{N}$ is an ideal, $B$ is a Boolean ring and a two-sided $R$-subgroup which is contained in the center of $R$, and $R \mathscr{N}^{2}=0$;

(iii) $R$ is self distributive and permutable.

Proof. Note that a right permutable near-ring is zero symmetric [4, Proposition 4.1].

Assume (i). As in the proof of Proposition 6.6, $\mathrm{gp}(\mathscr{I})=\mathscr{I}$ is a Boolean ring. Let $e \in \mathscr{F}$ and $k \in \mathscr{N}$; then $e k=e k e=e k^{3} e=0$. Therefore, part (ii) follows from Proposition 6.6 and Lemma 2.8 .

Assume (ii) and let $e_{i} \in B$ and $k_{i} \in \mathscr{N}$. Then

$$
\begin{aligned}
\left(e_{1}+k_{1}\right)\left(e_{2}+k_{2}\right)\left(e_{3}+k_{3}\right) & =\left(e_{1}+k_{1}\right)\left(e_{2}+k_{2}\right) e_{3}+\left(e_{1}+k_{1}\right)\left(e_{2}+k_{2}\right) k_{3} \\
& =e_{3}\left(e_{1}+k_{1}\right)\left(e_{2}+k_{2}\right)+\left(e_{1}+k_{1}\right) k_{3}\left(e_{2}+k_{2}\right) \\
& =e_{1} e_{2} e_{3}+\left(e_{1}+k_{1}\right) k_{2} k_{3}=e_{1} e_{2} e_{3} .
\end{aligned}
$$

Thus $R$ is self distributive and permutable. 
Assume (iii) and let $e, c \in \mathscr{I}$. From Lemma 6.7, $(e-c)^{2}=(e+c) e+$ $(e+c) c=e+e c+c e+c=e-c$. Hence $\operatorname{gp}(\mathscr{I})=\mathscr{J}$. Thus part (i) follows.

M. Petrich [20] has shown that a ring $R$ is self distributive if and only if $R=B \oplus \mathscr{N}$ is a ring direct sum, where $B$ is a Boolean ring and $\mathscr{N}^{3}=0$. Since a self distributive ring is permutable, Theorem 6.8 generalizes Petrich's result. However, even when $R$ is a commutative self distributive near-ring, for example [9, no. 29 on $\left.S_{3}\right], \mathscr{I}$ may not be a normal subgroup hence not an ideal. In [12, Theorem 11] C. Ferrero Cotti has attempted to generalize Petrich's theorem as follows: a distributive near-ring $R$ is self distributive if and only if $R=B+\mathscr{N}$ is a semidirect sum of a Boolean ring $B$ and a near-ring $\mathscr{N}$ such that $\mathscr{N}^{3}=0$. Theorem 6.8 generalizes her result since a distributive near-ring is permutable and since a direct sum of a Boolean ring and a nondistributive near-ring $\mathscr{N}$, where $\mathscr{N}^{3}=0$, is included under Theorem 6.8. Furthermore her statement is incomplete since the ring given in Lemma 5.7(iii) provides an example of an LSD but not self distributive ring $R$ where $R=B+\mathscr{N}$ is a semidirect sum of a Boolean ring and a nilpotent ring $\mathscr{N}$ such that $\mathscr{N}^{3}=0$. Our next result includes a condition which completes Ferrero Cotti's result.

COROLlARY 6.9. Let $R$ be a distributive near-ring. The following are equivalent:

(i) $R$ is self distributive;

(ii) $R=B+\mathcal{N}$ is a semidirect sum, where $B$ is a Boolean ring, $\mathscr{N}$ is an ideal, and $\mathscr{N}^{3}=0=B \mathscr{N}=\mathscr{N} B$.

Proof. That condition (i) implies condition (ii) follows from Lemma 6.7 (iv) and Theorem 6.8 .

The converse follows from an argument similar to that used for (ii) implies (iii) in Theorem 6.8.

In general, self distributive right permutable near-rings do not have the decomposition of Theorem 6.8 (see [9, nos. 9 and 37 on $\left.S_{3}\right]$ ). However, the right $R$-subgroups of the form $x R$ do have the decomposition of Theorem 6.8 .

COROLlaRY 6.10. Let $R$ be an LSD right permutable near-ring and $x \in$ $R$. Then $x R$ is a commutative near-ring and $x R=x^{3} R+\mathscr{N}(x R)$ is a semidirect sum (in $x R$ ), where $x^{3} R$ is a Boolean ring with identity $x^{3}$ and $\mathscr{N}(x R)$ is an ideal of $x R$.

Proof. By [4, Proposition 4.2], $x R$ is a commutative near-ring. From Theorem 6.8, $x R=B+\mathscr{N}(x R)$ is the desired semidirect sum and 
$B=\mathscr{I}(x R)$. Hence $x^{3} R \subseteq B$. Assume $b \in B$; then there exists $r \in R$ such that $b=x r$. Thus $b=b^{2}=x r x r=x^{3} r x r \in x^{3} R$. Consequently, $B=x^{3} R$.

THEOREM 6.11. Let $R$ be an LSD near-ring such that $\mathscr{I N}=0$, ce $\mathscr{I}=$ ec $\mathscr{I}$ for all $c, e \in \mathscr{I}$, and $R$ satisfies at least one of the following conditions

(i) $R$ has a reduced left ideal which is maximal among reduced left $R$ subgroups;

(ii) $e R$ is normal in $R^{+}$for all $e \in \mathscr{I}$.

Then $R=\mathbf{g p}(\mathscr{F})+\mathscr{N}$ is a semidirect sum, where $\mathbf{g p}(\mathscr{I})$ is a left ideal, $\mathscr{I} R \subseteq \mathbf{g p}(\mathscr{I})$, and $\mathscr{N}$ is an ideal. Furthermore, if condition (ii) holds or $R$ is zero symmetric, then $\mathbf{g p}(\mathscr{I})=\mathscr{I}$.

Proof. From Lemma $2.10, \mathscr{N}$ is an ideal. Let $e \in \mathscr{I}$ and $x, y \in R$. Then there exist $a, b \in \mathscr{I}$ such that $x e y=x e x^{3} x^{3} y=x^{4} e a y=e x^{3} b y$. Hence $e R$ is a left $R$-subgroup. Assume condition (i) and let $B$ be a left ideal which is maximal among reduced left $R$-subgroups. Suppose $e R \nsubseteq B$. By the maximality of $B$, there exists $0 \neq k \in \mathscr{N}$ such that $k=b+e s$, where $b \in B$ and $e s \in e R$. Then $0=e b+e s$. Therefore, $k=b-e b \in B \cap \mathcal{N}=0$, a contradiction. Consequently $e R \subseteq B$ for all $e \in \mathscr{I}$. By Lemma 2.9(iv), $B=\operatorname{gp}(\mathscr{I})$. If condition (ii) holds, let $B$ be a reduced left ideal which is maximal among reduced left ideals. An argument similar to the above will yield the result.

Examples illustrating the decomposition given in Theorem 6.11 can be found in [9, no. 22 on Klein four, nos. 57, 58, 60 on $\left.Z_{6}\right]$. Since these examples are not right permutable, Theorem 6.8 is not applicable. The next result gives a new characterization for self distributive rings.

COROLlaRY 6.12. Let $R$ be a ring. Then $R$ is self distributive if and only if $R$ is $L S D$ and $\mathscr{S N}=0$.

Proof. The corollary follows from Lemma 6.7, Theorem 6.8, and Theorem 6.11.

\section{Note Added in Proof}

From Theorem 1 of [D. J. Hansen and J. Luh, J. Austral. Math. Soc. 47 (1989), 103-107] and Proposition 4.3, it follows that every Boolean near-ring is self distributive. Hence Proposition 4.1 generalizes Theorem 1 of Hansen and Luh. 


\section{References}

[1] J. André, 'Non-commutative geometry, near-rings and near-fields,' Proc. Conf. Near-rings and Near-fields, edited by G. Betsch, pp. 1-14 (Amsterdam, 1987).

[2] J. Angerer and G. Pilz, 'The structure of near-rings of small order,' Lecture Notes in Computer Science No. 144, Computer Algebra, Marseille 1982, pp. 57-64, SpringerVerlag, 1982.

[3] G. Birkenmeier and H. Heatherly, 'Operation inducing systems,' Algebra Universalis 24 (1987), 137-148.

[4] G. Birkenmeier and H. Heatherly, 'Medial near-rings,' Monatsh. Math., 107 (1989), 89-110.

[5] G. Birkenmeier and $H$. Heatherly, 'Medial rings and an associated radical,' submitted.

[6] G. Birkenmeier and H. Heatherly, 'Medial near-rings: prime ideals and reduced nearrings,' submitted.

[7] G. Birkenmeier, H. Heatherly and T. Kepka, 'Rings with left self distributive multiplication,' submitted.

[8] G. Birkhoff, 'Subdirect unions in universal algebra,' Bull. Amer. Math. Soc. 50 (1944), 764-768.

[9] J. R. Clay, 'The near-rings on groups of low order,' Math. Z. 104 (1968), 364-371.

[10] J. R. Clay, 'Suggested direction for future research in near-ring theory,' Proc. Conf. Near-rings and Near-fields, edited by G. Ferrero and C. Ferrero Cotti, pp. 13-24 (San Benedetto del Tronto, 1981).

[11] S. Dhompongsa and J. Sanwong, 'Rings in which additive mappings are multiplicative,' Studia Sci. Math. Hungar. 22 (1987), 357-359.

[12] C. Ferrero Cotti, 'Sugli stems il cui prodotto è distributivo rispetto a se stesso,' Riv. Mat. Univ. Parma (3) 1 (1972), 203-220.

[13] H. Gonshor, 'On abstract affine near-rings,' Pacific J. Math. 14 (1964), 1237-1240.

[14] T. Kepka, 'Varieties of left distributive semigroups,' Acta Univ. Carolinae-Math. et Phys. 25 (1984), 3-18.

[15] S. Ligh, 'The structure of a special class of near-rings,' J. Austral. Math. Soc. Ser. A 13 (1972), 141-146.

[16] J. J. Malone, 'Near-rings with trivial multiplication,' Amer. Math. Monthly 74 (1967), $1111-1112$.

[17] J. J. Malone, 'D.G. near-rings on the infinite dihedral group,' Proc. Royal Soc. Edinburgh 78A (1977), 67-70.

[18] J. J. Malone, 'D. G. near-rings on $D_{2 n}, n$ even,' Worcester Polytechnic Inst. Math. Rep., 1978.

[19] J. D. P. Meldrum, Near-rings and their links with groups, (Pitman Research Notes No. 134, London, 1985).

[20] M. Petrich, 'Structure des demi-groupes et anneaux distributifs,' C. R. Acad. Sci. Paris Sér. $A-B 268$ (1969), A849-A852.

[21] G. Pilz, 'List of low order near-rings, along with some special properties,' Near-Ring Newsletter 2 (1980), 5-23.

[22] G. Pilz, Near-rings, (North-Holland, Amsterdam, 1983).

[23] D. Ramakotaiah and G. K. Rao, 'IFP near-rings,' J. Austral. Math. Soc. Ser. A 27 (1979), 365-370.

[24] R. Scapellato, 'On geometric near-rings,' Boll. Un. Mat. Ital. A (6) 2 (1983), 389-393.

[25] R. Scapellato, 'On autodistributive near-rings,' Riv. Mat. Univ. Parma (4) 10 (1984), 303-310.

[26] R. Scapellato, 'On geometric near-rings,' Proc. Conf. Near-rings and Near-fields, edited by G. Betsch, pp. 253-254 (Amsterdam, 1987).

[27] R. Scapellato, 'Strongly monogenic near-rings,' Arch. Math. 51 (1988), 476-480. 
[28] N. V. Subramanyan, 'Boolean semigroups,' Math. Ann. 148 (1962), 395-401.

[29] M.Willhite, Distributively generated near-rings on the dihedral group of order eight, (M. S. Thesis, Texas A\&M Univ., College Station, 1970).

[30] R. Yearby and H. Heatherly, 'The near-ring multiplications on Alt(4),' Near-Ring Newsletter 6 (1983), 59-73.

University of Southwestern Louisiana

Lafayette, Louisiana 70504

U.S.A. 